\title{
A Generalized Logistic Item Response Model Parameterizing Test Score Inappropriateness
}

\author{
Nancy L. Strandmer mond Robert L. Linu

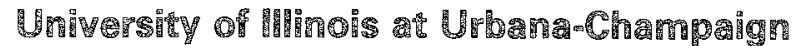

\begin{abstract}
The person response curve has been suggested as a possible model for test score inappropriateness (Lumsden, 1977, 1978; Weiss, 1973). The two-parameter person response curve proposed by Lumsden includes a person slope parameter but abandons the notion of differential item relatedness to the underlying trait. As an altemative, a generalized logistic model is considered that includes all item parameters of the threeparameter logistic model (Bimbaum, 1968). In addition to the usual person location parameter, the model has extra person parameters representing two possible characterizations of test score inappropriateness: a slope parameter indicating the degree to which a person responds differently to items of varying difficulty, and an asymptote parameter measuring a person's proclivity to engage in effective guessing or to omit items in the presence of partial information. To assess the model's feasibility, statistical comparisons were made between parameter estimates from data simulated according to the model and the original simulation parameters. The results seem encouraging, but additonal empirical study is needed before firm conclusions can be drawn.
\end{abstract}

An important psychometric problem that has received considerable attention in recent years concerns the accuracy and validity of individual test scores. Traditional indices, such as coefficient alpha and the standard error of measurement, provide descriptive information for the group but completely ignore any variation in measurement error

APPLIED PSYCHOLOGICAL MEASUREMENT

Vol. 11, No. 4, December 1987, pp. 355-370

(C) Copyright 1987 Applied Psychological Measurement Inc. 0146-6216/87/040355-16\$2.05 across persons. For the purpose of assessing the appropriateness of individual test scores, methods are needed that quantify measurement error at the level of the individual examinee. It is this problem that is considered here.

Tatsuoka and Linn (1983) have distinguished two general classes of techniques for use in detecting atypical patterns of item responses produced by individuals. The essential difference between the two approaches is that one is based on ordinary descriptive statistics derived from the persons $x$ items score matrix, whereas the other is modelbased. Examples of the first class of measures are the personal biserial correlation (Donlon \& Fischer, 1968), Sato's caution index (Tatsuoka, 1979), and the individual and norm consistency indices (Tatsuoka \& Tatsuoka, 1982). Model-based indices, all based on item response theory (IRT), include the appropriateness indices of Levine and his colleagues (Drasgow, 1982; Drasgow, Levine, \& Williams, 1984; Levine \& Drasgow, 1982; Levine \& Rubin, 1979), the squared standardized residual (Wright, 1977; Wright \& Stone, 1979), and the IRT indices derived by Tatsuoka and Limn (1983) as generalizations of the discrete descriptive statistics of Sato's student-problem curve theory (Tatsuoka, 1979). (Hullin, Drasgow, \& Parsons, 1983, have provided an excellent introductory discussion of the rationale for appropriateness measurement, with detailed descriptions of several of the techniques that have been implemented as well as some of the empirical results.) 
Except for the Gaussian model of Levine and Rubin (1979), all of the above IRT-based appropriateness measurement techniques conceptualize test score inappropriateness as the failure of the assumed test model to provide an adequate fit to the item responses of an individual examinee. An alvernate strategy is to model the most plausible types of test inappropriateness directly by including addicional person parameters within the assumed model. This approach represents an attempi to identify, explain, and individually quantify some of the sources contributing to the lack of model fit indexed by statistics such as the squared standardized residual (Wright, 1977; Wright \& Stone, 1979) and the IRT appropriateness indices (Drasgow, 1982; Drasgow et al., 1984 ; Levine \& Rubin, 1979).

It is this second modeling approach that was taken by Lumsden. Based on ideas suggested by Weiss (1973; Trabin \& Weiss, 1979, 1983; Vale \& Weiss, 1975), Lumsden proposed the concept of a person response curve as an alternative to the item response curve. According to Lumsden (1977, 1978,1980 ), it is much more realistic to localize measurement unreliability within the person rather than within the test item, as is customary. The model he favored, then, is that of a two-parameter person response curve of an examinee's expected item scores regressed onto item difficulty, which is the sole item parameter. Lumsden rejected the assumption of differential irem variances implied by a model that includes an item slope parameter because, in his opinion, it precludes the existence of test homogeneity, which is the primary assumption of all $\mathbb{I R T}$ models and is necessary to ensure local independence.

Lumsden (1980) and others (e.g., Andrich, 1978) have found it useful to conceptualize a person-item encounter using Thurstone's law of comparative judgment (see Torgerson, 1958). When the variances of both person and item propensity distributions are possibly nonzero and are not necessarily identical, this formulation leads to a model that is similar in spirit to the one introduced in this paper. Both models include parameters for the variances of the two propensity distributions, but they differ with respect to the way in which those pa- rameters mathematically combine. Hence, the Thurstone formulations can be regarded as cornpetitors to the model presented here.

When models such as the Gausssian model, the person response curve, the Thurstone-like model, and the generalized logistic model of this paper are fit to observed data, they automatically produce one or more values containing information about the validity of individual test scores, thens permitting hypotheses to be made regarding plawsible sources of invalidity when if is detected. These models use important information that has always been available in test protocols but which has heretofore been ignored. Tapping this information could result in wiser usage of measuring instruments, because only scores of acceptable validity would be used in practical applications of test data.

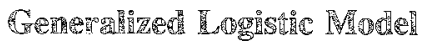

The model proposed here is a generalized item response model that includes both a person parameter and an item parameter; their multiplicative combination determines the slope of the irem and person response functions. An additional parameter of this model characterizes differential proclivities of examinees to guess the correct answer when they do not know it. The expected item score of person $g$ on item $i$ is expressed by the equation

$P_{g i}=\left(c_{i}+\gamma_{g}\right)+\frac{1-\left(c_{i}+\gamma_{g}\right)}{1+\exp \left[0 a_{i} \alpha_{g}\left(\theta_{g}-b_{i}\right)\right]}$,

where $\theta$ and $b$ are the locations of the person and item on the underlying trait continuum, $a$ and $a$ are person and item parameters that together moderate the slope of the regression function, and $\gamma$ and $c$ are person and item parameters that adjust the lower asymptote of the function to account for individual guessing behavior on particular tesî items. The constant $D$ is ser equal to 1.702 in order to maximize iesemblance of the logistic function to a normal ogive.

Four constraints on the model parameters are needed in order to ensure their uniqueness. Peth the origin and unit of the location parameters, th

Downloaded from the Digital Conservancy at the University of Minnesota, http://purl.umn.edu/93227. May be reproduced with no cost by students and faculty for academic use. Non-academic reproduction requires payment of royalties through the Copyright Clearance Center, http://www.copyright.com/ 
and $b$, must be specified. This can be accomplished by requiring that $\mu_{\theta}=0$ and $\sigma_{\theta}=1$, which is the same convention that is usually applied for the twoand three-parameter logistic models. Even with these two constraints, the units of the slope parameters, $\alpha$ and $a$, remain anidentifed. After multiplying one of them by $\sigma_{\theta}$ or by multiplying them by complementary factors of $\sigma_{\theta}$, it is still possible to multiply values of $\alpha$ by any positive factor $k$ and multiply values of $a$ by its multiplicative inverse, $1 / k$, without affecting model fit.

This fact implies that the units of $a$ and $a$ must be fixed by an additional arbitrary constraint; it is suggested that the sum of squares of the $\alpha$ parameter values be required to equal $N$. A further lack of uniqueness in the model involves the asymptote parameters, $\gamma$ and $c$. If a positive constant, $k$, in the range $(0,1)$, such that $k \geqslant \operatorname{Min}(\gamma)$ and $k \leqslant 1-$ $\operatorname{Max}(c)$, is subtracted from all values of $y$ and is added to all values of $c$, model fit is again unchanged; use of the smallest value satisfying the stated conditions is suggested, which will almost certainly be $\operatorname{Min}(\gamma)$ in practice.

The suggested set of constraints is consistent with the current practice of guaranteeing the uniqueness of model parameters by imposing reasonable but arbitrary conditions on the person parameters. With these four constraints, or alternate ones having equal merit, the problems of the uniqueness and identifiability of parameter estimates are resolved, and the parameters should be estimable, given adequate data.

This model is a generalization of the unidimensional logistic item response models that are in current use. If $\gamma$ is required to assume a constant value in the range $(0,1)$, it is in effect absorbed into each item asymptote parameter, $c$. In a similar manner, if $\alpha$ is required to be a negative constant across all persons, it is absorbed into $a$, with a minus sign attached. With these changes the model reduces to the three-parameter logistic model (Bimbaum, 1968). Requiring either $c$ or $\gamma$ to be a constant (or constraining both of them at 0 ) effectively eliminates one or both of those parameters from the model; likewise, constraining $a$ and/or $a$ to positive and negative constant values, respectively, also simplifies the model. Any of the 15 combinations of slope and asymptote parameters can be removed in one of these ways. With the additional possibility of requiring both $c$ and $\gamma$ to be nonzero constants, there are then a total of 19 possible submodels of Equation 1.

The additional person parameters in this generalized model are an explicit attempt to represent directly two possible characterizations of test score inappropriatemess. A unique feature of the model is that it defines a continuous regression surface rather than a simple regression curve. For a fixed person, $\alpha, \theta$, and $\gamma$ are constant across all items, while $a, b$, and $c$ are random variables; for a fixed item, the roles of parameters and random variables are reversed. In other words, whether each of these triples is a vector of parameters or of random variables depends on whether it is the person or the item that is considered fixed. When the item is fixed, the equation represents an item response function; when the person is fixed, it describes a person response function.

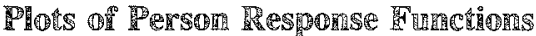

Whether Equation 1 is momentarily being considered a person or an item response function, it is impossible to depict it in graphic form; doing so would require four dimensions. The best that can be accomplished is to fix all parameters and all but one of the random variables, and then plot probability as a function of values of the remaining random variable. Because examination of such plots can be helpful in clarifying the meaning of this model, a sequence of person response runction plots is provided in Figures 1,2, and 3. Only plots with $a$ and $b$ on the horizontal axis are shown; when probability is regressed onto $c$, the result is always linear, and corresponding functions differ only im slope and/or separation.

In Figure 1, the probability of a correct response for selected values of the three person parameters is plotted as a function of $a$. The value of $c$ is also constant, so that the three functions on the same pair of coordinate axes differ from each other only in the value of $b$, which is either $-1,0$, or 1 . The 
Fighinge 1

Person Response Functions Regressed Onto $a$ With a Separate Curve for Each of Three Values of $b_{i}$ $\left(c_{i}=.10\right.$ and $\left.\gamma_{g}=.10\right)$

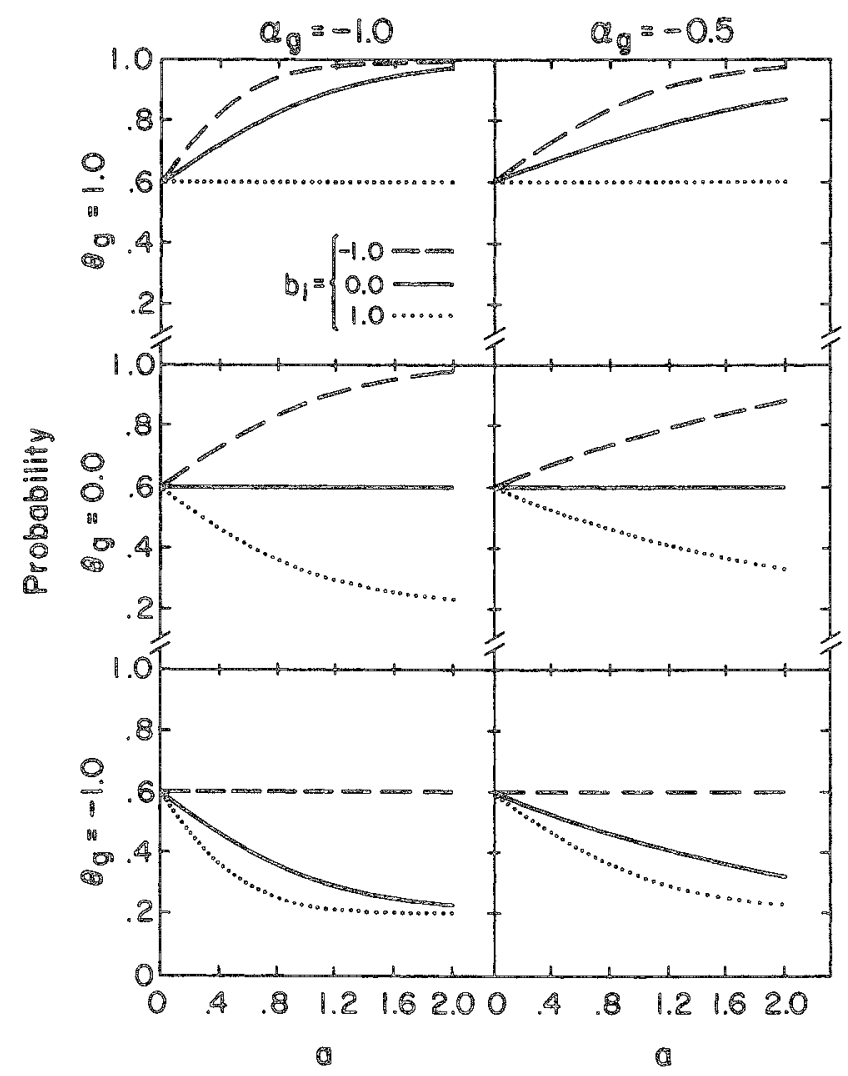

highest curve always corresponds to the lowest value of $b$, and the lowest curve always corresponds to the highest value. Because $b$ is the item difficulty parameter, this merely reflects the fact that easier irems have higher probabilities of correct response than do items of greater difficulty.

In Figure 2, the probability of a correct response for selected values of the three person parameters is plotted as a function of $a$, as in Figure 1 . The value of $b$ is also constant, so that the three functions on the same pair of coordinate axes are alike except for the value of $c$, which is either $.00, .05$, or .10. In each plot, the highest curve corresponds to the highest value of $c$ and the lowest curve cor- responds to the lowest value, illustrating how the item asymptote parameter reflects the possibility of effective guessing on a test item.

The plots in any one column of Figures 1 and 2 differ from each other only in the value of $\theta$, the person location parameter, which equals 1,0 , and -1 for rows 1, 2, and 3, respectively. Because $\theta$ is the usual ability parameter, it is not surprising that increases in its value are accompanied by increases in the value of $P_{g i}$. The plots in any one row of Figures 1 and 2 differ from each other only in one respect. The person slope parameter, $\alpha$, is -1.0 in column 1 and -.5 in column 2 . Comparing the two columns of Figures 1 and 2 shows 
W

Person Response Functions Regressed Onto a With a Separate Curve for Each of Three Valmes of $c_{i}$

$$
\left(b_{i}=0.0 \text { and } \gamma_{g}=.10\right)
$$

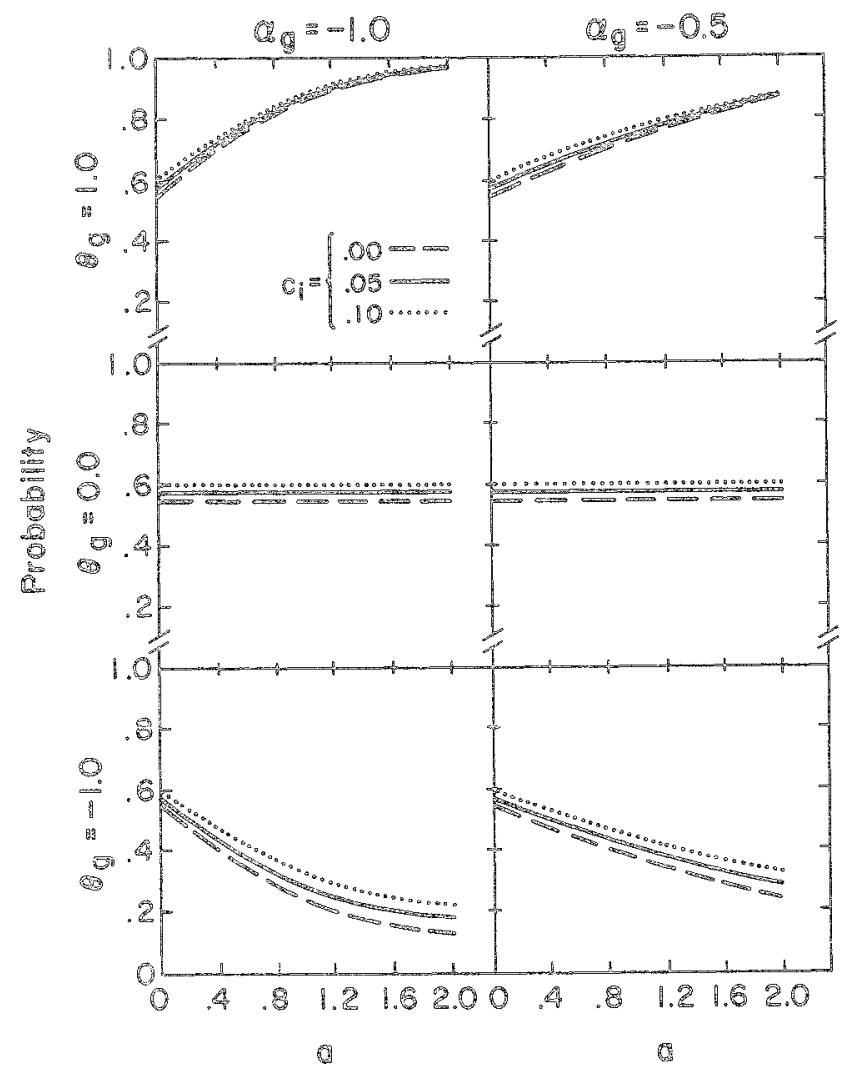

that the higher he value of $\alpha$, the faster $\mathbb{F}_{g i}$ increases or decreases with increases in $a$, so that a intuences the function's rate of change.

Each plot in Figures 1 and 2 inlustrates the erfect of $a$, the item slope parameter, represented by the horizontal axis. If $a_{i}=0, a, b$, and $b$ have no influence on $\mathbb{F}_{\text {gis }}$, the separation at $a_{i}=0$ in each plot of pigure 2 is caused by differences in $c$. Wherever $a_{i}=0$ or $\mathbb{O}_{g}-b_{i}=0$, then $F_{g i}=\left(c_{i}\right.$ $\left.+y_{g}\right)+\left[1-\left(c_{i}+y_{g}\right)\right] / 2$. When $a_{g}-b_{i}>0$ the curves approach an upper asymptote of 1 as the value of $a$ increases, and when $b_{g}-b_{i}<0$ they approach a lower asymptote of $c_{i}+\gamma_{g}$ as the value of $a$ increases. In Figure 2 , when $\theta_{g}-b_{i}>0$ the amounit of separation between curves decreases as the value of $a$ increases, and when $\theta_{g}-b_{i}<0$ the amounit of separation increases.

Figure 3 gives two plots of probability as a function of $b$. Except for negative slopes and asymptotes at the high end of the horizontal axis, the plots do not differ from ordinary three-parameter logistic item response functions. Increasing the value of 9 translates a curve to the right, leaving its shape intach, whereas decreasing $\theta$ by the same value moves the curve the same distance to the left. From the left panel of Figure 3 it can be seen how increases in a cause the curve to have a steeper slope. The right panel of Figure 3 shows how changes in 


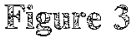

Person Response Functions Regressed Onto $b$ With a Separace Curve for Each of Three Values of $a_{i}$ and $c_{i}$, Respectively

$$
\left(\alpha_{g}=-1.0, \theta_{g}=0.0 \text {, and } \gamma_{g}=.10\right)
$$

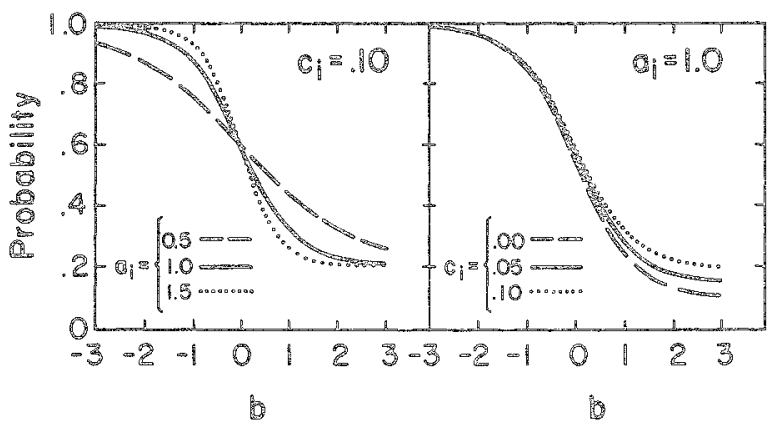

c cause a nonlinear change in the curve, increasing or decreasing probabilities at higher levels of $b$ but having trivial effecrs at the lower end of the scalle. Changes in $\gamma$ would produce similar results. The slope of each curve in Figure 3 is proportional to the product of $a_{i}$ and $\alpha_{g}$, and the lower asymptote is simply $c_{i}+\gamma_{g}$.

As the plots in Figures 1, 2, and 3 illustrate, lower absolute values of $\alpha$ and $a$ lessen an item's success at differentiating among ability levels as well as a person's usefulness in providing information about comparative item difficulties. The asymptote parameters are most infuential at low values of $\theta$ and at high values of $b$, where they infifate the probability of comeciness. The two parameters combine additively, and therefore do not interact.

Analogous views of item response functions have been onitued but can easily be imagined. Plots with $\alpha$, the person slope paraneter, on the horizontal axis resemble those in Figures 1 and 2 . Wher probability is plotted as a function of $\theta$, the plots are most like those for $b$ in Figure 3, and when $y$ is on the horizontal axis the plots are limear, just as they are for $c$. The differences between corresponding item and person response function plots result from the opposite signs of the item and person slope parameters and the reversed relationships of the location parameters to probability (i.e., the greater the value of $\theta$, the more likely it is that the response will be correct, but higher values of $b$ predict lower probabilities of correctness).

The urility of this generalized logistic model depends on the ability of iterative parameter estimation procedures to obtain sufficiently accurate estimates of the person parameters, as well as on the model's ability to capture some of the sources of individual differences contributing to the appropriateness of test scores. An attempt has been made to estimate the parameters of the model using simulated data with what appears to be relatively good success.

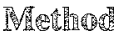

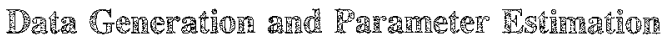

Data were generated to simulate the responses of 2,195 persons to 111 test items. The location parameters, $A$ and $b$, were both drawn from the standard normal distribution, truncated in the range $(-3,3)$. All other parameters were taken from uniform distributions. The ranges for the slope parmeters, $a$ and $a$, were $(-1.5,-.5)$ and $(.5,1.5)$, respectively, and the lower asymptote parameters, $\gamma$ and $c$, were both drawn from the range $(.025,15)$.

The simulated itern score data were submitted to a parameter estimation routine designed to es- 
tinnate all 3 a $+3 N$ paramerers of the model, where ris the mumber of simulated items and $N$ is the number of simnlated examinees. Item scores of only the first 1 , 000 simulated examinees were included in the callbration sample, so that a total of $3(111+1000)=3,333$ parameters were estimated.

Ar iterative parameter estingation procedure was to be used which, considering the number of parameters, was expected ro be comparanvely expensive everi by RT standards. For this reason, it Was important that the initial estimates provided to the routine be as accurate as possible, assuming that the true parmeters were unknown. Initial estimates for the location and slope parameters were computed as functions of the item and person biserial correlations, as outlined by Lord (1980, pp. 33-34). Although Lord's rationale does not stricty apply to this model, it was nevertheless accepted as a reasonable approach. Estimates for $\gamma$ and $c$ were initially set at .10. An alfemate strategy for deriving initial parameter estimates would have been to obtain them by applying LOGIST (Wood, Wingersky, \& Lord, 1976$)$ to the persons $X$ îems score matrix, and then to its trarspose. Neither procedure is exactly suited to this task; therefore the less costly alternative was used.

The person and îem parameters were esênated by a cyclical, modified maximum likelihood procedure similar to that used by LoGIST (Lord, 1980; Wood et a․, 1976). During the first part of each major cycle, person paraneters were reestimated while consideving the currentitem parameter estrmates as the true values; after all person paraneters were reescimated, the item paraneters were updated, whith the latest person parameter estimates treated as true parameters. Each person and item was recalibrated by a separate call to the optimization algorithm. This estimation procedure relies heavily on the assunption of local independence of items and persons. It is consistent with current practice for estimating the parameters of two-way item response models for which conditional estimation is not possible. It is not known whether such a procedure converges to a global optimum for a two-way model, but the results generally appear to be good.
The IMSL (1982) subroutine ZXMIN Was employed to detemime the set of parameter estimates that would optimize the megative log likelhood corresponding to each person or test item. The algorithm is based on the Harwell library rourine VA10A (Fletcher, 1972). It uses a quasi-Newton estimation method and is highly regarded as an efficient, accurate, and readily available tool for that purpose (Michael Levine, personal commumication, Fall 1982). Even though ZXMN assumes the existence of the gradient vector and the Hessim manix, ir does not require the user to supply formulas for them; therefore, the only explicit function it reguires is the function that is to be optimizized.

Before each call to ZXMIN, some of the current parameter escimates that were to undergo modifcation were transformed by monotonic functions designed to impose constraints on the resulting estimates. The inverse transformations were applied berore each evaluation of the negative log likellhood to obviate having to adjust the likelihood fuction by the Jacobian matrix associated with the particular transformations that were used. (The inverse transfonmations were applied again after leaving ZXMIN, although, in retrospect, this extra step was probably avoidable.) Imposing bounds on the parameters using such transformations places restrictions on the parameter space, making the es促证ation procedure only a pseudo-maximm likelihood method. This modification can be conceptualized as a Rayesian-like procedure that is based on infomed expectations about the true palameter space. Its primary purpose is to ensure that all estimated parameters fall within reasonable ranges. (This procedure was suggested by Michael Levine, personal communication, Fall 1982.) The function

$x_{1}=\ln \left(\frac{2-a^{*}}{a^{*}-.3}\right)$

and its inverse,

$a^{*}=\frac{1.7}{1+\exp \left(x_{1}\right)}+.3$

were used, with $a^{*}=a_{i}$ or $a^{*}=\left|\alpha_{g}\right|$, depending on whether item or person parameter estimates were 
being updated. If $a^{*}>2-\delta$ or $a^{*}<.3+\delta$, where $\delta=.0001$, hit was replaced by that value before $x_{1}$ was computed. Likewise, if the new value of $x_{1}$ supplied by ZXMIN was less than In (.00005) or greater than In $(17,000)$, it was replaced by that value to preverat underflow and overifow errors. These values were derived by rounding the bracketed part of Equation 2 after it was computed using values of $2-8$ and $.3+8$. For $a$, the value of $a^{*}$ from Equation 3 was also multiplied by -1 . The slope parameters, $a$ and $\alpha$, were in this way bounded by $(.3001,1.9999)$ and $(-1.9999$, $-.3001)$, respectively. In a similar manner, bounds of $(.0001, .1499)$ were imposed on the estimates for $c$ and $y$ using the equation

$x_{3}=\ln \left(\frac{.15-c^{*}}{c^{*}}\right)$

and its inverse,

$c^{*}=\frac{.15}{1+\exp \left(x_{3}\right)}$.

In this case $c^{*}=c_{i}$ or $c^{*}=\gamma_{g}$ unless $c^{*}>.15-\delta$ or $c^{*}<\delta$, when it was set to the closer of these two values. As before, $\delta=.0001$. If $x_{3}<\ln (.0007)$ or $x_{3}>\ln (2,500)$, the value of $x_{3}$ was reset to the value in the inequality. The location parameters were unbounded, so that $x_{2}=b^{*}$, where $b^{*}=b_{i}$ or $b^{*}=\theta_{g}$, depending on which parameters were being updlated.

Due to the complexiry of the estimation problem, some compromises were made in choosing specific options for the estimation algorithm. In particular, ZXMIN permits several options for determining initial values of the Hessian matrix. Originally the program specifed that ZXMIN cormpute an estimate of the Hessian matrix. When this procedure failed to enact changes in the parameter estimates, the Hessian marrix estimated by ZXMEN was constrained to be diagonal. Because this procedure provided updates that continually improved from one cycle to the next, is was retained. An alternative that was not tried would have been to estimate the second derivatives independently of the minimization subroutine and then supply the Hessian matrix to ZXMMNN. 咕 is not known whether such a procedure would have resulted in better estimates and/or faster convergence.

Parameter estimation reguaned a considerable mumber of continuation runs. At the end of each mun, location and slope estimates were nomalized by the following transformations:

$\hat{\theta}_{g}^{*}=\frac{\hat{\mathbb{B}}_{g}-\hat{\hat{\theta}}_{\theta}}{\hat{\mathbb{G}}_{\theta}}$,

$\hat{b}_{i}^{*}=\frac{\hat{\hat{b}}_{i}-\hat{\hat{\omega}}_{\theta}}{\hat{\sigma}_{0}}$,

$\hat{Q}_{g}^{*}=\left(\hat{\mathbb{O}}_{\theta}\right)^{1 / 2} \hat{Q}_{g}$,

and

$\hat{a}_{i}^{*}=\left(\hat{\sigma}_{\theta}\right)^{1 / 2} \hat{a}_{i}$.

The parameters $\hat{c}_{i}$ and $\hat{\gamma}_{g}$ were left unchanged.

Parameter estimation was considered complete when the changes in the person and item likelihoods from one cycle to the next were both less than of equal to .001. A very strict convergence criterion was used for calls to ZXMiN so that ail possible values of the original parameters would be estimated with acceptable accuracy, despite the use of the nonlinear transformations that were substituted as input to the estimation subroutine. Thus, it was no surprise that the betwenn-cycles criterion was satisfied first.

The estimation procedure required 69 cycles to reach convergence. Twice during estimation, the parameter estimates began to diverge; whenever this happened, the problem was relieved by backing up to the previously saved estimates and resstimating the updates using a lower value for the maximum number of calls to ZXMHN. A sell-contained algorithm designed especially for this particular estimation task might be expected to achicve more accurate estimates with much greater efficiency. The purpose of this study, however, was to examine the feasibility of this model rather than to develop an optimal estimation procedure.

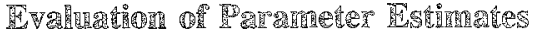

Several different types of descriptive statistics were computed to assess the accuracy of the final parameter estimates by comparing them with ohe 
true parameters from which the data were generated. Before some of the computrations were made, additional transomations were carred ont on both the simulated and estimated parameters. In particular, the slope parameter escimats were ransformed to have equa mean sums of squares and the asymptote paraneiers were transformed io equalize their means. Diferent constants from those used for the simulated parameters were required for the estimated parameters. All transfomations were consistent with the conditions stated above and were of lithe conseguence; they were used sinmply becanse these indeterninacies exist in the model, and it was known that a and a were simulated rom distribarions with egual dispersions and that $y$ gnd $c$ were simulated from distributions will equal means. In the remaining text, all equations will be written without the asterisks; the symbols should then be regarded as either the nornalized estimates or the nomalized estimates arfer submission to this additional set of remsformations, depending on the context.

Correlotions. The simplest of the descripative statistics are product-moment coprelations berween the estrimated and simulated parmeters. Becanse all valid parmener ransfomations ane linear, they have no effect on the values of these correlation coeficieries.

Root mean squared and mean absolute differences. To assess the accuracy of the asymptote parameter estimates, two unsefal statistics are the root mean squared difference (RMSD) beween the oniginal and estinated parameters,

RMSD $=\left[\frac{\sum_{i=1}^{n}\left(x_{i}-\hat{x}_{i}\right)^{2}}{n}\right]^{1 / 2}$,

and the mean absolute difference (MAD),

$\mathrm{MAD}=\frac{\sum_{i=1}^{n}\left|x_{i}-\hat{x}_{i}\right|}{R}$.

Unlike the correlation coefficient, low values of these siatistics signify good int. Also, note that they are not invarian with respect to most valid ransformations of the paramerters; the only exception is when the constant used to transform the asymp- tote parameters is the same for simulated and escimated parameters.

Bics stutistes. Th addition to the criterion that simulated and esimated parmmeters to highly correlated, it is also desirable that the estimates be unbiased in a statistical sense, so that, on the avmage, parmeters are neither over mor underestimated. The customary seatistic for this purpose is

Dias $=\frac{\sum_{i=1}^{n}\left(x_{i}-\hat{x}_{i}\right)}{n}$.

Whenever parameter transformations can affect values of the RMSD and MAD statistics, they can also affect the computer bias.

\section{W}

Tor purposes of evaluating 解e information provided by he paraneters on the type and extent of individual dofrences and iten properties, the above comparisons are suitable. However, it is a iso important to examine how successfully the data are fit in the callibration sample, as well as how satisfactorily the model operotes across the entire range of probable person and iter parmeters. To answer such gluestions, if is necessary to compare the true and estimated probabilities generated by the model. Wote that any valid transfonmations carried on on the sirmulated and estimated parameters do not afm fect these statistics.

Roor mean squared differences. The first comparison 㪽ing probabilicies involved compulation of RisDs beween probabilites derived asing the simulated and estimated parameters. Any resuls from this statistic can be expected to be samplespecific, in the sense that they gre generalizable only to a population with similar distributions of the person and item parameters. 直 addition, each item (person) staistic reflects entor mot only in the parameters of the item (person) it represents, but also in the estimated person (item) parameters; hence is is not possible to pirpoint the exact camses of inaccuracy. The distributena characteristics of the RMSD are unknown, but it can at least be intenoreted by comparison with irs perfect baseline of 0 and

Downloaded from the Digital Conservancy at the University of Minnesota, http://purl.umn.edu/93227. May be reproduced with no cost by students and faculty for academic use. Non-academic reproduction requires payment of royalties through the Copyright Clearance Center, http://www.copyright.com/ 
can also be assed to make ordinal comparisons among iterns.

Alhough computing MADS would also have been appropriate, this was not done. Squaring deviations increases the relative contribution of outliers, so that MADS would generally be lower than the RMSD siatistics.

Root integrals. Another statistic similar to the RMSD is the root integral

$\mathbb{R l}_{i}=\left(\iint_{\mathbb{a}^{\alpha}} \int_{\theta_{\gamma}} \Delta p_{i} \partial \gamma \partial 0 \partial \alpha\right)^{1 / 2}$

and its analogue,

$\mathbb{R I}_{g}=\left(\iint_{a} \int_{b} \Delta p_{c} d c \partial b \partial a\right)^{1 / 2}$,

where $\Delta P_{i}=P_{i}(\alpha, \theta, y)-\hat{P}_{i}(a, \theta, y)$ for Equation 13 and $\Delta P_{g}=P_{g}(a, b, c)-\hat{P}_{g}(a, b, c)$ for Equation 14. Unlike the observed RMSDs, these statistics implicitly consider discrepancies in all areas of the range of integration as being of equal significance.

Equations 13 and 14 were calculated using probabilities computed from the simulated and estimated parameters of this study after all parameter transformations had been camied out as described above. The IMSL (1982) subroutine DMLIN, which integrates functions of several random variables by mumerical methods, was used. The functions were integrated across the same intervals from which the parameters were originally drawn:

$$
\begin{aligned}
& a:(.5,1.5) \\
& a:(-1.5,-.5) \\
& b, 0:(-3,3) \\
& \text { and } \\
& c, \gamma:(.025, .15) \text {. }
\end{aligned}
$$

The RMSDs and the root integrals produce different orderings of the persons and items. This is because of the dependence of the Rirstos on the parameter distributions, and the dependence of the root integrals on the ranges of integration. No comparisons between the sets of values of the two sta tistics can logically be made, therefore, becalse they provide two distinct kinds of information.

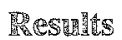

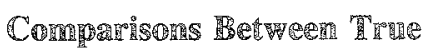

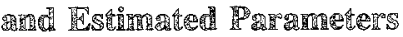

All computed statistics used to assess the parameter estimates are displayed together in Table 1 . The bias statistics computed before and after transfomation differed enough so that both sets of values ane reported.

Corpelations. Each of the correlations between estimated and tue parameters is statistically significant at $p<.05$. However, the suitability of statistical rests on these correlations is doubtiul. Except for the two location parameters, which were sampled from nomal distributions and estimated from the simulated data with no bounds imposed, the parameters do not meet the necessary assumptions of normality and homoscedasticity. In addition, the parameters are interdependene, having been estimated logether from a common set of data.

Root mean squared and mean absolute differences. The correlation coefficient is a more suitable statistic for the location and slope parameters; therefore, only RMSD and MAD values for the asymptote parameters are given in Table 1. Paraneter transformation effected a negligible change in

Table 1

Statistical Comparisons Between True and Estimaced Parameters

\begin{tabular}{lcccccc}
\hline Statistic & $a$ & $b$ & $c$ & $\alpha$ & $\theta$ & $y$ \\
\hline Correlation & .781 & .967 & .178 & .552 & .873 & .166 \\
RMSD & -- & -- & .039 & -- & - & .046 \\
MAD & -- & -- & .033 & -- & -- & .037 \\
Bias & & & & & & \\
$\quad \begin{array}{l}\text { Normalized } \\
\text { Transfomed }\end{array}$ & -.223 & -.016 & -.005 & .241 & -.028 & .002 \\
& -.196 & -.016 & -.002 & .268 & -.028 & -.001 \\
\hline
\end{tabular}

Downloaded from the Digital Conservancy at the University of Minnesota, http://purl.umn.edu/93227. May be reproduced with no cost by students and faculty for academic use. Non-academic reproduction requires payment of royalties through the Copyright Clearance Center, http://www.copyright.com/ 
only one vallue, hence only the statistics computed ising the original, nomalized parameter estimates are reported. Because of the bounds imposed during parameter estimation, statistical tests are inappropriate. However, all of these values can be evaluated with respect to the range of $c$ and $y$, which was $(.00,15)$; by that standard they are relatively good.

Bias statistics. Because the location parameters were sampled from the standard normal distribution and the person location parameter estimates were transformed by Equation 6, the discrepancy of this statistic from ofor person location parameters reflects only the inexactmess in drawing a finite sample from an infinite population. Bias statistics for item location and for person and irem slope and asymptote parameters carny more meaning. The size of the bias statistic for the item location parameter estimates is trivial. Person and item slope parameters both tended to be biased outward, and the bias was usually more extreme for persons than it was for items. If upper bounds had not been enforced, this effect might well have been even more drastic. The asymptote parameter estimates, though relatively inexact, seem to be unbiased despite the imposition of bounds.

The statistics cited thus far indicate that item parameters were estimated better than person pa- rameters; $b$ ut because the distributions from which corresponding parameters were drawn were idenhical, this cam easilly be explained by the unequal sample sizes. The location parameter estimates are quire accurate, slope estimates are moderately good, and estimates of asymptotes are only mediocre.

\section{CoIHPari มำ}

Root mear squared differences. A RMSD statistic was calculated for each item, averaging across persons, and for each person, averaging across items. For items, the average RMSD was .066, the standard deviation was .011, and the skewness was .961; comparable values for persons were .062, .023, and 1.231 , respectively. The five highest and low est values for items and persons are shown in $\mathrm{T}$ ables 2 and 3 . It is quite encouraging that the value of the RMSD exceeds. 10 for just $2 / 111=1.8 \%$ of the items and for only $72 / 1000=7.2 \%$ of the persons. However, it must be kept in mind that these values are somewhat reduced by the ideal condition that the person and item location parameter distributions were nomal with equal means and variances. In other words, the test was centered exactly where the most infomation was meeded. Among the tabled values, high RMSDs are associated with

Table 2

The 5 Highest and 5 Lowest

Iten Root Hean Squared Differences $(x=111$ Itens)

\begin{tabular}{|c|c|c|c|c|c|c|c|}
\hline Iten & a & $\hat{\mathrm{a}}$ & $b$ & $\hat{b}$ & $c$ & $\overrightarrow{\mathrm{c}}$ & RUSD \\
\hline \multicolumn{8}{|c|}{ Highest } \\
\hline 14 & .508 & 1.253 & .844 & .417 & .073 & .037 & .1134 \\
\hline 109 & .825 & 1.270 & .209 & .018 & .049 & .100 & .1041 \\
\hline 68 & 1.082 & 1.257 & .280 & .018 & .110 & .098 & .0986 \\
\hline 36 & 1.432 & 1.108 & 1.379 & 1.155 & .032 & .077 & .0893 \\
\hline 96 & .806 & 1.189 & .096 & .036 & .027 & .100 & .0836 \\
\hline \multicolumn{8}{|l|}{ Lowest } \\
\hline 9 & .677 & .915 & -.629 & -.276 & .054 & .094 & .0472 \\
\hline 80 & .616 & .775 & .100 & .167 & .085 & .081 & .0465 \\
\hline 35 & .527 & .752 & -.427 & -.188 & .069 & .099 & .0440 \\
\hline 107 & .540 & .640 & .368 & .233 & .054 & .032 & .0438 \\
\hline 20 & .545 & .643 & .251 & .148 & .135 & .086 & .0405 \\
\hline
\end{tabular}

Downloaded from the Digital Conservancy at the University of Minnesota, http://purl.umn.edu/93227. May be reproduced with no cost by students and faculty for academic use. Non-academic reproduction requires payment of royalties through the Copyright Clearance Center, http://www.copyright.com/ 
Table 3

The 5 IIghest and 5 Towest

Person Root Mean Squared Differances

$(N=1,000)$

\begin{tabular}{|c|c|c|c|c|c|c|c|}
\hline Petson & $\alpha$ & $\hat{\alpha}$ & $\theta$ & $\hat{\theta}$ & $\gamma$ & $\hat{y}$ & MHSD \\
\hline \multicolumn{8}{|l|}{ Highest } \\
\hline 111 & -.835 & -.972 & -.965 & $=.043$ & .032 & .200 & .2235 \\
\hline 604 & -1.460 & -1.997 & -.525 & .041 & .210 & .080 & .1591 \\
\hline 251 & -1.479 & -1.867 & -1.050 &. .956 & .098 & .015 & .1572 \\
\hline 426 & -1.405 & -.600 & -1.224 & -1.992 & .114 & .029 & .1484 \\
\hline 42 & -1.306 & -.537 & -.030 & .122 & .084 & .094 & .1472 \\
\hline \multicolumn{8}{|l|}{ Lowest } \\
\hline 260 & -.695 & -.911 & .750 & .464 & .059 & .101 & .0265 \\
\hline 441 & -.768 & -1.057 & -.043 & .020 & .064 & .069 & .0264 \\
\hline 391 & $=.577$ & $=.852$ & .246 & .153 & .072 & .085 & .0282 \\
\hline 174 & -.518 & -.548 & .398 & .325 & .104 & .104 & .0250 \\
\hline 319 & -.690 & -.820 & -.133 & -.005 & .124 & .098 & .0228 \\
\hline
\end{tabular}

extreme values of the true slope parameter about half of the time. Also, the true valle of $b$ is usually positive and the true value of $\theta$ is usually negative for the tabled entities with high valnes of RMSD.

Root ivtegrals. For items, the average root integral was .064 , the standard deviation was .029, and the skewness was .610; corresponding values for persons were .071, 038 , and .834 , respectively. The five highest and lowest values of these item and person root integrals are shown in Tables 4 and 5. Of the item root integrals, $12 / 111=$ $10.8 \%$ are greater than $.10 ; 198 / 1000=19.8 \%$ of the person root integrals exceed that value. The root integrals tend to be especially high when both the location and the slope parameters are estimated poorly. When both of those parameters are estimated well, that fact is reflected in a low root integral value.

The irem and person root integrals appear to serve the purpose for which they were intented; the righ values in Tables 4 and 5 are for items and persons with particularly badly estimated paramoters. The fact that the mean item root integral is lower than the mean for persons agrees with expectation based on the greater accuracy of item parameter estimates. This result stands in contrast to that for the fwo RivisD means.

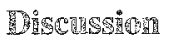

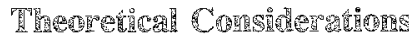

The ideas incorporated into the generalized logistic model presented here are mot entirely new. The person asymptote parameter is meant to recognize knowin individual dirferences in the terdency to guess at itenas that are 100 difficullt, rather than to omit then or to omit items in the presence of partial knowledge. Lord and Novick (1968, p. 304) have used the term "omissiveness" for this particular response style trait. The person slope parameter comes directly from recent thinking involving the concept of a person response curve (Lumsden, 1977, 1978, 1980; Trabin \& Weiss, 1979, 1983; Vale \& Weiss, 1975; Weiss, 1973).

A useful theoretical comparison involves the contras berween the Gaussian model of Levine and Rubin (1979) and the generalized logistic model of this study. Levine and Rubin were very adamant about their preference always to consider their model as that of an irem response function with nxed item parameters and a single ramion variable representing the individual's trait standing. At each item administration a value of $a_{g i}$ is sampled from a distribution that is assumed to be normal with mean to and variance $\sigma_{0}^{2}$ that are estimable from the data.

Downloaded from the Digital Conservancy at the University of Minnesota, http://purl.umn.edu/93227. May be reproduced with no cost by students and faculty for academic use. Non-academic reproduction requires payment of royalties through the Copyright Clearance Center, http://www.copyright.com/ 
Table 4

The 5 fighest and 5 Lowest Item Root Integrals $(n=111)$.

\begin{tabular}{|c|c|c|c|c|c|c|c|}
\hline Itema & $\mathrm{a}$ & $\hat{a}$ & $b$ & $\hat{\mathrm{b}}$ & $c$ & $\hat{c}$ & $\mathrm{RI}$ \\
\hline \multicolumn{8}{|c|}{ Highest } \\
\hline 85 & .717 & 1.198 & -1.195 & -.466 & .087 & .146 & .1458 \\
\hline 14 & .508 & 1.253 & .844 & .417 & .073 & .037 & .1433 \\
\hline 11 & 1.428 & 1.710 & -1.334 & -.895 & .033 & .100 & .1427 \\
\hline 56 & .760 & .770 & -2.134 & -1.603 & .053 & .149 & .1173 \\
\hline 86 & .737 & 1.084 & $=.989$ & -.466 & .058 & .128 & .1173 \\
\hline \multicolumn{8}{|c|}{ Lowest } \\
\hline 7 & 1.343 & 1.141 & -2.187 & -2.309 & .136 & .150 & .0250 \\
\hline 62 & .686 & .892 & 1.399 & 1.135 & .097 & .150 & .0227 \\
\hline 66 & .905 & .956 & .254 & .238 & .037 & .079 & .0185 \\
\hline 46 & .614 & .669 & .275 & .109 & .077 & .092 & .0170 \\
\hline 5 & 1.363 & 1.473 & 1.884 & 1.812 & .079 & .088 & .0088 \\
\hline
\end{tabular}

Table 5

The 5 Pighest and 5 Lowest Person Root Integrals $(\mathrm{N}=1,000)$

\begin{tabular}{|c|c|c|c|c|c|c|c|}
\hline Peresor & $\alpha$ & $\hat{\alpha}$ & $\theta$ & $\hat{\theta}$ & $\gamma$ & $\hat{\gamma}$ & $R I$ \\
\hline \multicolumn{8}{|c|}{ Highest } \\
\hline 507 & -1.335 & -.771 & -1.760 & -5.442 & .029 & .081 & .2734 \\
\hline 455 & -.727 & -.352 & -1.916 & -9.491 & .056 & .133 & .2323 \\
\hline 434 & -1.189 & -1.997 & -1.117 & -.278 & .082 & .009 & .2289 \\
\hline 250 & -.742 & $=1.997$ & 1.287 & .443 & .063 & .099 & .2009 \\
\hline 886 & $=.671$ & -1.370 & -1.343 & -.466 & .067 & .029 & .1887 \\
\hline \multicolumn{8}{|l|}{ Lowest } \\
\hline 775 & $=.791$ & -.796 & -.210 & -.163 & .077 & .078 & .0069 \\
\hline 405 & -.620 & -.602 & .560 & .469 & .111 & .103 & .0065 \\
\hline 497 & -.714 & -.713 & .000 & .003 & .086 & .097 & .0065 \\
\hline 48 & -.631 & -.662 & .712 & .694 & .092 & .099 & .0045 \\
\hline 746 & -1.419 & -1.393 & .353 & .307 & .100 & .100 & .0037 \\
\hline
\end{tabular}

The variance (or its square root) then functions as the appropriateness index for the examinee. If the item in the model presented above is also assumed to be fred, then the random variables in it are $\alpha$, Q. and $y$.

These random variables are assumed to be sampled only once for each test administration rather than each time an additional item is administered, as in the Gaussian model. Their underlying sampling distributiorns are across testing occasions rather than item administrations. Interest is centered only on their current values, hence no assumptions regarding distributional forms need be made. The accuracy of the estimates of $\mathbb{a}, \theta$, and $\gamma$ is dependent on the adequacy of sampling from a homogeneous universe of possible test items, whereas the Gaussian model considers the test as a fixed entity, with mo sampling having taken place.

Ultimately, the choice of a test model must depend on the plausibility of its assumptions regard- 
ing statistical sampling processes, which can be assessed by goodness-of-fit criteria that take into account model complexity. Considerations involving necessary sample sizes and test lengths as well as the expense of model fiting may mean that the most plausible models can rarely, if ever, be applied. However, advances in computing technology make the application of models such as the Gaussian model and the generalized logistic model more feasible.

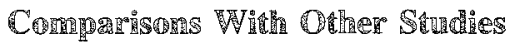

As with any linear or nonlinear model containing a large number of parameters, the possibility of acceptable estimation using a finite sample of data is an initial question that must be addressed by empirical monte carlo techniques, even before its applicability to real data can be examined; such was the purpose of this study. One way in which the results can be put into perspective is by comparing them with findings from similar studies, keeping in mind that any variations must be interpreted cautiously in light of the differences in models, sample sizes, test lengths, parameter sampling distributions, computational procedures, and convergence criteria.

Hulin, Lissak, and Drasgow (1982) and Ree (1979) investigated the accuracy of simultaneous parameter estimation for the three-parameter logistic model as it is achieved by the LOGIST algorithm (Wood ef all., 1976). The correllations between simulated and estimated parameters common to the three-parameter and generalized logistic models for all three studies are displayed in Table 6. Ree (1979) obtained correlations that consistently exceed the values obrained here, perhaps because of differences in sample size, model complexity, and parameser distributions. The difference in model complexity might also explain the higher correlation for $\hat{\theta}$ achieved by Hulin et al. (1982). Their lower correlations for item parameters are somewhat unexpected, but differences in sampling distributions and estimation procedures probably affected the results. Hulin et al. also reported root mean squared difference statistics, but differences in computational method preclude meaningful comparisons with the values obtained here.

\section{Conchelesions}

Much more work needs to be done before this model can be applied. The next step in its development is to improve the parameter estimation algorithm as much as possible, and then apply it to real data. If the fit to real data with large values of $n$ and $N$ is worse than that provided by the threeparameter logistic model, then the full model with all three person parameters and three item parameters should be discarded.

If this model does give a better fir to real data, then the next logical step would be to test whether the added parameters provide reliable information on individual differences relating to test score appropriateness. A study similar to that carried out by Drasgow (1982) would be ideal. Only if the extra person parameters offer reliable information surpassing or supplementing that provided by simpler techniques can the use of this model be justified. Even if the use of this general model is unsupported after further study, it is still possible that one or more of its submodels, for example the submodel that assumes the person asymptotes are

Table 6

Corredations Between True and Estimated Paranders

\begin{tabular}{lrrrrrr} 
Study & N & n & a & b & c & $\theta$ \\
\hline Present study & 1000 & 111 & .781 & .967 & .178 & .873 \\
Hulir et a1. (1982) & 1000 & 60 & .543 & .939 & $=-$ & .898 \\
Ree (1979) & 2000 & 80 & .827 & .975 & .379 & .965 \\
\hline
\end{tabular}

Downloaded from the Digital Conservancy at the University of Minnesota, http://purl.umn.edu/93227.

May be reproduced with no cost by students and faculty for academic use. Non-academic reproduction requires payment of royalties through the Copyright Clearance Center, http://www.copyright.com/ 
all equal to on to some other constant, might provide a good model for some measurement applications.

\section{Referereries}

Andrich, D. (1978). Relationships between the Thursione and Rasch appronches to item scaling. Applied Psychological Measurement, 2, 451-462.

Bimbaum, A. (1968). Some latent trait models and their use in infening an examince's ability. In F. M. Lord \& M. R. Novick, Statistical theories of mertal test scores. Reading MA: Addison-Wesley.

Donlon, T. F., \& Fischer, F. E. (1968). An index of an individual's agreement with group-determined item difficulties. Educational and Pychological heasuremernt, 28, 105-113.

Drasgow, $\mathrm{F}$. (1982). Choice of test model for appropriateness measurement. Applied Psychological Measurement, $6,297-308$

Drasgow, F. Levine, M. V., \& Williams, E.A. (1984). Appropriateness measurement with polychotomous item response models and standardized indices (Measurement Series 84-1). Champaign IL: University of IIlinois.

Fletcher, R. (1972). FORTRAN Subroutines for minimization by musid-Newon methods (Report R7125). Harwell, England: Atomic Energy Research Estabiishment.

Hulin, C. L., Drasgow, F., \& Parsons, C. K. (1983). tem response theory: Application to psychological measurement. Homewood 埴: Dow Jones-Irwin.

Mulin, C. I., Lissak, R. I., \& Drasgow, T4. (1982). Recowery of two- and three-parameter logistic item characteristic curves: A monte carlo study. Applied Psychological Measurement, 6, 249-260.

MMSE Library (1982, 9th ed.). Houston TK: Intemational Mathematical and Statiscical Libraries.

Levine, M. V., \& Drasgow, F. (1982). Appropriateness measurement: Review, critique, and validating studies. Brivish Joutral of Wathematical and Statistical Psychology, 35, 42-56.

Levine, M.V., \&ubin, D. B. (1979). Measuring the appropriateness of multiple-choice test scores. Jowmal of Educcutional Statistics, 4, 269-290.

Lord, F. M. (1980). Applications of item response theory to practical testing problems. Hillsdale NJ: Eribamm.

Lord, F. M., \& Novick, M. R. (1968). Statistical theories of mental test scores. Reading MA: AddisonWesley.

Lumsden, J. (1977). Person reliabiliry. Applied Psychologica Measurement, $1,477-482$.

Lumsden, I. (1978). Tests are perfectly reliable. British Jourtal of Mathematical and sratistical Pychology, 31, $19-26$.
Lumsden, J. (1980). Variations on a theme by Thurstone. Applied Psychological Measurement, 4, $1-7$.

Ree, M. I. (1979). Estimating item characteristic curves. Applied Psychological Measurement, 3, 371-385.

Tatsuoka, K. K. K., \& Linn, R. L. (1983). Indices for detecting unusual pattems: Links between two general approaches and potential applicarions. Applied Psy. chological Measurement, 7, 81-96.

Tatsuoka, K. K., \& Tatsuoka, M. M. (1982). Detection of abcrant response pattems and their effect on dimensionality. Journal of Edrecational Statistics, 7, 215231.

Tatsuoka, M. M. (1979). Recent psychomentic developments in Japan: Engineers tackle educational measurement problems. ONR - Tokyo Science Bulletin, 4 , $1-7$.

Torgerson, W. S. (1958). Theory and methods of scating. New York: Wiley.

Trabin, T. E., Wciss, D. I. (1979). The person response curve: Fit of individuals to item chat acteristic curve models (Research Report 79-7). Minneapolis: University of Minnesota, Department of Psychology. (NTTES No. AD-A080 933)

Trabin, T. E., \& Weiss, D. I. (1983). The person response curve: Fir of individuals to item response theory models. In D. J. Weiss (Ed.), New horizons in testing: Latent trait test theory and computerized adaptive testing (pp. 83-108). New York: Academic Press.

Vale, C. D., \& Weiss, D. I. (1975). A study of computeradministered stradaptive ability testing (Research Report 75-4). Minneapolis: University of Mimnesota, Department of Psychology. (NTIS No. AD-A018758)

Weiss, D. J. (1973). The stratifed adaptive computerized ability test (Research Report 73-3). Minneapolis: University of Minnesota, Department of Psychology. (NTIS NO.AD-768 376)

Wood, R. L., Wingersky, M. S., \& Lord, F. M. (1976). LOGIST-A computer program for estimating examinee ability and item characteristic curve parameters (Research Memorandum 76-6). Princeton NJ: Educational Testing Service.

Wright, B. D. (1977). Solving measurement problems with the Rasch model. Jowrnal of Educational Measurement, 14, 97-116.

Wright, B. D., \& Stone, M. H. (1979). Best test design. Chicago: Mesa Press.

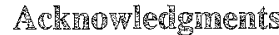

This article is based on the master's thesis of the first author, conducted while a predocioral trainee in the Quantitative Methods Program of the Deparment of Psychology, University of thinois at Upbanch-Chompaigr. The research was supported in part by the Al-

Downloaded from the Digital Conservancy at the University of Minnesota, http://purl.umn.edu/93227. May be reproduced with no cost by students and faculty for academic use. Non-academic reproduction requires payment of royalties through the Copyright Clearance Center, http://www.copyright.com/ 
cohol, Drug Abuse, and Mental Health Adwinistration, National Research Service Award No. MH14257. The awhors thank Charles $\mathbb{L}$. Hultin for suggesting some of the data analyses and commenting on earlier drafts of this anticle, and Michael $V$. Levine for suggesting a useful parameter estimation strategy.

\section{A}

Send requests for reprints or furher information to Nancy L. Strandmark, Deparment of Psychology, Universiry of llinois, $603 \mathrm{E}$. Daniel St., Champaign IL 61820 , U.S.A.

Downloaded from the Digital Conservancy at the University of Minnesota, http://purl.umn.edu/93227.

May be reproduced with no cost by students and faculty for academic use. Non-academic reproduction requires payment of royalties through the Copyright Clearance Center, http://www.copyright.com/ 